\title{
Analysis of Beams and Thin Plates Using the Wavelet-Galerkin Method
}

\author{
Rodrigo Bird Burgos, Marco Antonio Cetale Santos, and Raul Rosas e Silva
}

\begin{abstract}
The use of wavelets has become increasingly popular in the development of numerical schemes for the solution of partial differential equations (PDEs), especially for problems with local high gradient. In this work, the Galerkin Method has been adapted for the direct solution of differential equations in a meshless formulation using Daubechies wavelets and Deslauriers-Dubuc interpolating functions (Interpolets). This approach takes advantage of wavelet properties like compact support, orthogonality and exact polynomial representation, which allow the use of a multiresolution analysis. Several examples based on typical differential equations for beams and thin plates were studied successfully.
\end{abstract}

Index Terms-Wavelets, interpolets, wavelet-galerkin method, beam on elastic foundation, thin plates.

\section{INTRODUCTION}

The use of wavelet-based numerical schemes has become popular in the last two decades. Wavelets have several properties that are especially useful for representing solutions of partial differential equations (PDEs), such as orthogonality, compact support and exact representation of polynomials of a certain degree. Their capability of representing data at different levels of resolution allows the efficient and stable calculation of functions with high gradients or singularities [1].

Compactly supported wavelets have a finite number of derivatives which can be highly oscillatory. This makes the numerical evaluation of integrals of their inner products difficult and unstable. Those integrals are called connection coefficients and they appear naturally when applying a numerical method for the solution of a PDE. Due to some properties of wavelet functions, these coefficients can be obtained by solving an eigenvalue problem.

Working with dyadically refined grids, Deslauriers and Dubuc (1989) obtained a new family of wavelets with interpolating properties, later called Interpolets [2]. Unlike Daubechies' wavelets [3], Interpolets are symmetric, which is especially interesting in numerical analysis.

The use of wavelets as interpolating functions in numerical schemes holds some promise due to their multiresolution properties. The approximation of the solution can be

Manuscript received May 14, 2014; revised July 20, 2014

R. B. Burgos is with the State University of Rio de Janeiro (UERJ), Department of Structures and Foundations, RJ, Brazil (e-mail: rburgos@ig.com.br).

M. A. Cetale Santos is with Fluminense Federal University (UFF), ISIS Group, Department of Geology and Geophysics, RJ, Brazil (e-mail: marcocetale@id.uff.br).

R. R. Silva is with Pontifical Catholic University of Rio de Janeiro, Department of Civil Engineering, RJ, Brazil (e-mail: raul@puc-rio.br). improved by increasing either the level resolution or the order of the wavelet used.

Several examples were used for validating the proposed method. First, a beam with a concentrated load was used to test the method's ability to capture discontinuities. As a second example, critical buckling loads for axially loaded beams on elastic foundation with several boundary conditions were obtained. The method was then applied for the static analysis of thin plates showing excellent performance in 2-D models. Results are presented and compared with analytical values, when available.

\section{WAVELET THEORY}

Multiresolution analysis using orthogonal, compactly supported wavelets has been successfully applied in numerical simulation. Wavelet basis are composed of two kinds of functions: scaling functions $(\varphi)$ and wavelet functions $(\psi)$. The two combined form a complete Hilbert space of square integrable functions. The spaces generated by scaling and wavelet functions are complementary and both are based on the same mother function [4].

\section{A. Daubechies Wavelets}

In the following expressions, known as the two-scale relation, $a_{k}$ are the scaling function filter coefficients and $N$ is the Daubechies wavelet order.

$$
\varphi(x)=\sum_{k=0}^{N-1} a_{k} \varphi(2 x-k), \quad \psi(x)=\sum_{k=0}^{N-1}(-1)^{k} a_{N-1-k} \varphi(2 x-k)(1)
$$

In general, there are no analytical expressions for wavelet functions, which can be obtained using iterative procedures like (1). In order to comply with the requirements of orthogonality and compact support, wavelets present, in general, an irregular fractal-like shape. Fig. 1 shows Daubechies scaling function of order $N=4$.

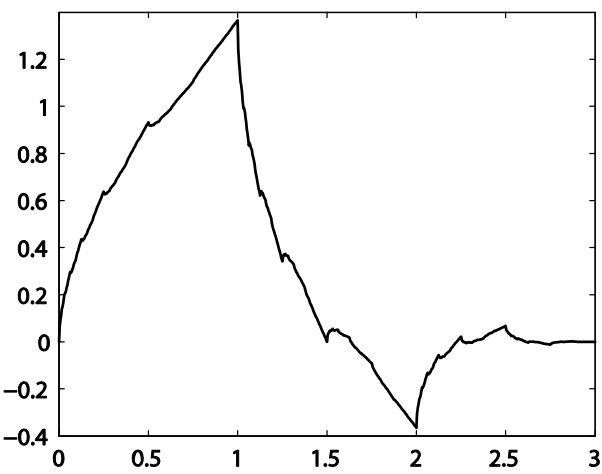

Fig. 1. Daubechies scaling function of order $N=4$. 


\section{B. Deslauriers-Dubuc Interpolets}

The term interpolet was first used by [5] to designate wavelets with interpolating characteristics. The basic characteristics of interpolating wavelets require that the mother scaling function satisfies the following condition [6]:

$$
\varphi(k)=\delta_{0, k}=\left\{\begin{array}{ll}
1, & k=0 \\
0, & k \neq 0
\end{array}, \quad k \in \mathbb{Z}\right.
$$

The filter coefficients $c_{k}$ for Deslauriers-Dubuc scaling function of order $N$ can be obtained by an autocorrelation of the same order Daubechies' filter coefficients.

$$
\begin{aligned}
& \varphi(x)=\sum_{k=1-N}^{N-1} c_{k} \varphi(2 x-k) \\
& c_{k}=\sum_{m=0}^{N-1} a_{m} a_{m-k}
\end{aligned}
$$

Interpolets satisfy the same requirements as other wavelets, specially the two-scale relation, which is fundamental for their use as interpolating functions in numerical methods. Fig. 2 shows the Interpolet IN4. Its symmetry and interpolating properties are evident.

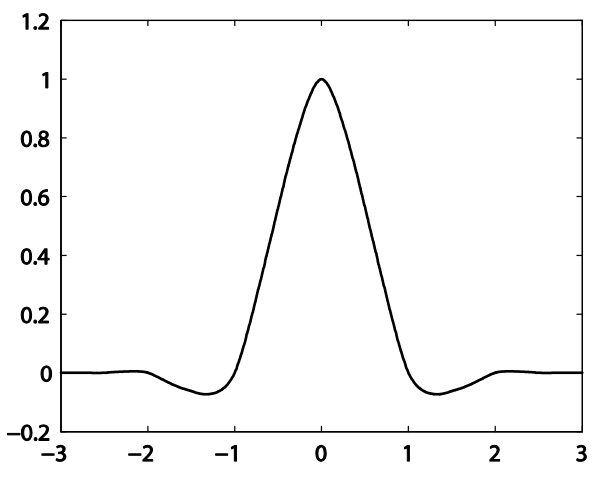

Fig. 2. Interpolet IN4 scaling function.

\section{Connection Coefficients}

Assuming that a function $f(x)$ is approximated by a series of interpolating scale functions, the following may be written:

$$
f(x)=\sum_{k} \alpha_{k} \varphi(x-k)
$$

The process of solving a differential equation (DE) requires the calculation of the inner products of the basis functions and their derivatives $\left(d_{1}, d_{2}\right)$. These inner products are defined as connection coefficients and are given by:

$$
\Gamma_{i, j}^{d_{1}, d_{2}}=\int \varphi^{\left(d_{1}\right)}(x-i) \varphi^{\left(d_{2}\right)}(x-j) d x
$$

The values for the limits of the integral in (5) depend on which method is used to impose boundary conditions. In this work, the limits are given by $\left[0,2^{\mathrm{m}}\right]$, where $m$ is the wavelet level of resolution. This method allows the use of Lagrange multipliers to deal with boundary conditions, similarly to what is usually done in a meshless scheme [7]. Connection coefficients at level $m$ can be obtained through the calculation at level 0 thus avoiding its recalculation while increasing the level of resolution. Wavelet dilation and translation properties allow the calculation of connection coefficients within the interval $[0,1]$ to be summarized by the solution of an eigenvalue problem based only on filter coefficients [8].

$$
\begin{aligned}
& \left(\boldsymbol{P}-\frac{1}{2^{d_{1}+d_{2}-1}} \boldsymbol{I}\right) \boldsymbol{\Gamma}^{\boldsymbol{d}_{\mathbf{1}}, d_{2}}=0 \\
& P_{i, j: k, l}=a_{k-2 i} a_{l-2 j}+a_{k-2 i+1} a_{l-2 j+1}
\end{aligned}
$$

Since (6) leads to an infinite number of solutions, there is the need for a normalization rule that provides a unique eigenvector. This unique solution comes with the inclusion of the so-called moment equation, derived from the wavelet property of exact polynomial representation [9].

$$
\begin{aligned}
& \sum_{i, j} M_{i}^{k} M_{j}^{k} \Gamma_{i, j}^{d_{1}, d_{2}}=\frac{(k !)^{2}}{\left(k-d_{1}\right) !\left(k-d_{2}\right) !\left(2 k-d_{1}-d_{2}+1\right)} \\
& M_{i}^{j}=\frac{1}{2^{j+1}-2} \sum_{k=0}^{j}\left(\begin{array}{l}
j \\
k
\end{array}\right) i^{j-k} \sum_{l=0}^{k-1}\left(\begin{array}{l}
k \\
l
\end{array}\right) M_{0}^{l}\left(\sum_{i=0}^{N-1} a_{i} i^{k-l}\right)
\end{aligned}
$$

\section{WAVELET-GALERKIN METHOD}

The numerical solution of DE's is one of the possible applications of the wavelet theory. The Wavelet-Galerkin Method (WGM) results from the use of wavelets as interpolating functions in a traditional Galerkin scheme. In the following sections, the WGM will be applied to solve typical DE's for structures like beams and plates.

\section{A. Axially Loaded Beam on Elastic Foundation}

The homogeneous DE for a beam with rigidity EI on a Winkler-type foundation of stiffness $c$ subjected to an axial load $P$ is given by:

$$
E I \frac{\partial^{4} v}{\partial \xi^{4}}+P \frac{\partial^{2} v}{\partial \xi^{2}}+c v=0
$$

Stiffness $(k)$, foundation $(b)$ and geometry $(g)$ matrices can be obtained by substituting the displacement $v$ by a series of interpolating functions. Non-dimensional coordinates $\xi$ within the interval [ $\left[\begin{array}{ll}0 & 1\end{array}\right]$ are used in wavelet space, leading to the subsequent expressions:

$$
\begin{aligned}
& (k+b+P g) \alpha=\mathbf{0} \\
& k_{i, j}=E I \int_{0}^{1} \varphi_{i}^{\prime \prime}(\xi) \varphi_{j}^{\prime \prime}(\xi) d \xi=E I \Gamma_{i, j}^{2,2} \\
& b_{i, j}=c \int_{0}^{1} \varphi_{i}(\xi) \varphi_{j}(\xi) d \xi=c \Gamma_{i, j}^{0,0} \\
& g_{i, j}=\int_{0}^{1} \varphi_{i}^{\prime}(\xi) \varphi_{j}^{\prime}(\xi) d \xi=\Gamma_{i, j}^{1,1}
\end{aligned}
$$


As done in the traditional Finite Element Method (FEM), critical loads and buckling modes can be obtained by solving an eigenvalue problem of the form:

$$
\left\{\left[\begin{array}{cc}
\boldsymbol{\Gamma}^{2,2}+c \boldsymbol{\Gamma}^{0,0} & -A^{T} \\
-\boldsymbol{A} & \mathbf{0}
\end{array}\right]-\frac{P}{E I}\left[\begin{array}{cc}
\boldsymbol{\Gamma}^{1,1} & \mathbf{0} \\
\mathbf{0} & \mathbf{0}
\end{array}\right]\right\}\left\{\begin{array}{l}
\boldsymbol{\alpha} \\
\boldsymbol{\lambda}
\end{array}\right\}=\left\{\begin{array}{l}
\mathbf{0} \\
\mathbf{0}
\end{array}\right\}
$$

where the matrix $\boldsymbol{A}$ is associated with boundary conditions and $\lambda$ is a vector of Lagrange multipliers. The main difference in relation to the FEM is that the unknowns in vector $\boldsymbol{\alpha}$ are interpolating coefficients of the basis functions instead of nodal displacements. In fact, there is no need to establish nodal coordinates.

\section{B. Thin Plate}

The bending of a thin plate with thickness $t$ consisting of a material with Young's modulus $E$ and Poisson's ratio $v$ is modeled by the following DE:

$$
\begin{aligned}
& D\left(\frac{\partial^{4} w}{\partial \xi^{4}}+2 \frac{\partial^{4} w}{\partial \xi^{2} \partial \eta^{2}}+\frac{\partial^{4} w}{\partial \eta^{4}}\right)=q(\xi, \eta) \\
& D=\frac{E t^{3}}{12\left(1-v^{2}\right)}
\end{aligned}
$$

Displacement $w(\xi, \eta)$ is modeled using bi-dimensional wavelets, which are products between one-dimensional wavelets:

$$
w(\xi, \eta)=\sum_{i} \sum_{j} d_{i j} \phi(\xi-i) \phi(\eta-j)
$$

As done in a traditional Galerkin approach, Eq. (16) is substituted in the DE and integrated, leading to a system of equations which contains the wavelets' connection coefficients [10].

$$
\begin{aligned}
\boldsymbol{k} \boldsymbol{d} & =\boldsymbol{f} \\
\boldsymbol{k} & =D\left[\boldsymbol{\Gamma}^{\mathbf{0 0}} \otimes \boldsymbol{\Gamma}^{\mathbf{2 2}}+v\left(\boldsymbol{\Gamma}^{\mathbf{2 0}} \otimes \boldsymbol{\Gamma}^{\mathbf{0 2}}+\boldsymbol{\Gamma}^{\mathbf{0 2}} \otimes \boldsymbol{\Gamma}^{\mathbf{2 0}}\right)+\right. \\
& \left.+\boldsymbol{\Gamma}^{\mathbf{2 2}} \otimes \boldsymbol{\Gamma}^{\mathbf{0 0}}+2\left(1-v^{2}\right) \boldsymbol{\Gamma}^{\mathbf{1 1}} \otimes \boldsymbol{\Gamma}^{\mathbf{1 1}}\right], \\
\boldsymbol{f} & =\left[\int_{0}^{1} q(\eta) \boldsymbol{\Phi}^{\mathrm{T}} d \eta\right] \otimes\left[\int_{0}^{1} q(\xi) \boldsymbol{\Phi}^{\mathrm{T}} d \xi\right]
\end{aligned}
$$

The symbol $\otimes$ indicates Kronecker product. The system is solved for vector $\boldsymbol{d}$ using the stiffness matrix $\boldsymbol{k}$ and force vector $\boldsymbol{f}$ provided by (13) and imposing essential boundary conditions with Lagrange multipliers as done before for one-dimensional DE's.

\section{EXAMPLES}

\section{A. Simply Supported Beam}

Fig. 3 shows a simple example of a beam subjected to a concentrated load at its midpoint. This example was formulated in order to verify the ability of the wavelet method to deal with singularities, since the load generates a discontinuity in the shear force diagram.

This example is easily solved by dividing the beam in two elements and applying the load as a nodal force. In this work, since degrees of freedom don't have a fixed position, the load must be transformed into the wavelet space:

$$
q(\xi)=P \delta\left(\xi-\frac{1}{2}\right) \rightarrow f_{j}=P \varphi\left(\frac{1}{2}-i\right)
$$

The example was solved using the IN8 Interpolet at different levels of resolution and the results for bending moment and shear force diagrams are shown in Figs. 4 and 5. It is clear that higher levels of resolution are necessary in order to capture the singularity that occurs where the load is applied. Nevertheless, results are considerably good, since the solution is obtained in wavelet space and no discretization was performed. The discontinuity in the slope of the bending moment is captured even for a low level of resolution.

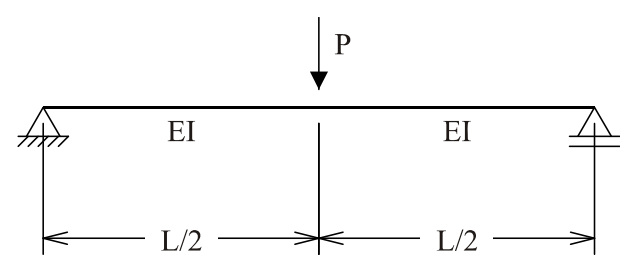

Fig. 3. Beam with concentrated load.

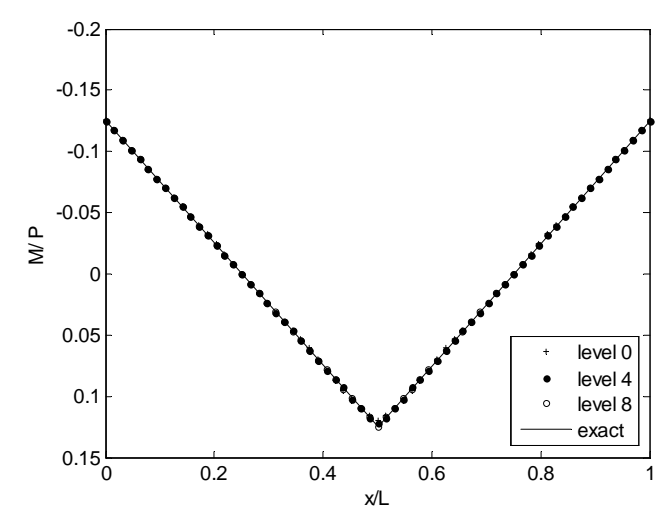

Fig. 4. Bending moment using IN8.

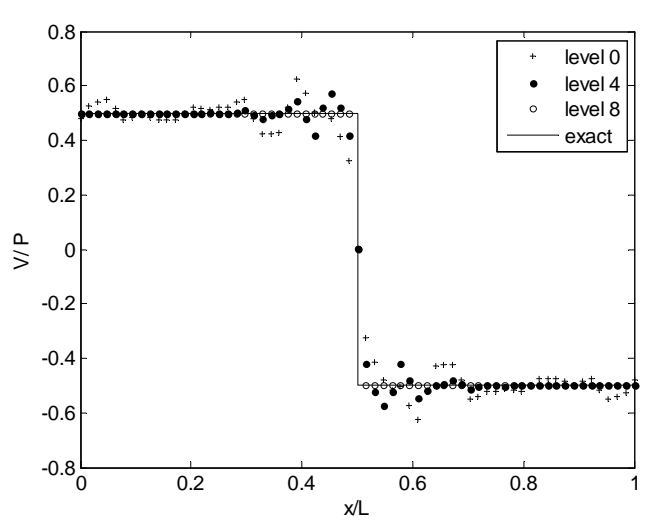

Fig. 5. Shear force using IN8.

\section{B. Axially Loaded Beams on Elastic Foundation}

\section{1) Simply supported}

A simply supported beam on elastic foundation is shown in 
Fig. 6. Reference [11] presents some possible values for critical loads of a simply supported beam on elastic foundation:

$$
P=\frac{\pi^{2} E I}{L^{2}}\left(n^{2}+\frac{c L^{4}}{n^{2} \pi^{4} E I}\right), \quad n=1,2, \ldots
$$

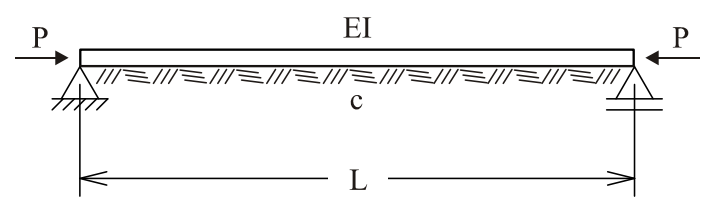

Fig. 6. Axially loaded simply supported beam on elastic foundation.

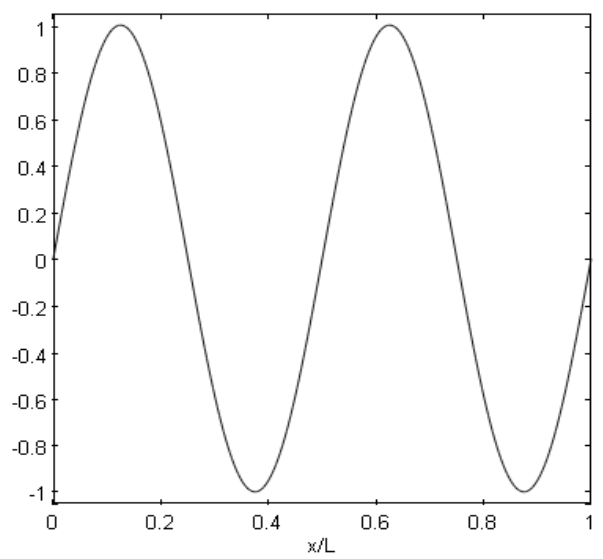

Fig. 7. Buckling mode of an infinitely long simply supported beam.

Depending on the stiffness of the foundation and the length of the beam, different values of $\mathrm{n}$ can give the smallest critical load. For example, the transition between $n=1$ and $n$ $=2$ occurs when $c=4 \pi^{4} E I / L^{4}$.

For $c \gg E I / L^{4}$, the critical load is the same obtained to guarantee a periodical solution, $P=2 \sqrt{c E I}$. This is normally due to a rigid foundation or an infinitely long beam.

Table I presents the results obtained for the first and second critical loads considering $c=\pi^{4} E I / L^{4}$. Results are shown for Daubechies (DB) and Interpolet (IN) wavelets and are compared with exact values.

TABLE I: RESUlTS FOR A SIMPLy SUPPORTED BEAM ON ELASTIC FOUNDATION

\begin{tabular}{c|c|c|c|c|c|c|c}
\hline \multicolumn{7}{c}{ Critical Loads $\left(E I / L^{2}\right)$} \\
\hline \multirow{3}{*}{ mode } & \multirow{3}{*}{ exact } & \multicolumn{3}{c}{ DB10 } & \multicolumn{3}{c}{ IN4 } \\
\cline { 3 - 8 } & & \multicolumn{2}{|c|}{ level of resolution } & \multicolumn{3}{c}{ level of resolution } \\
\cline { 3 - 8 } & 0 & 4 & 8 & 0 & 4 & 8 \\
\hline 1 & 19.739 & 19.744 & 19.739 & 19.739 & 20.219 & 19.741 & 19.739 \\
\hline 2 & 41.946 & 59.193 & 42.004 & 41.946 & 48.925 & 42.069 & 41.946 \\
\hline \hline
\end{tabular}

TABLE II: RESUlTS FOR A SIMPLY SUPPORTED BEAM ON ELASTIC FOUNDATION WITH $C=4 \pi^{4} E I / L^{4}$

Critical Loads $\left(E I / L^{2}\right)$

\begin{tabular}{c|c|c|c|c|c|c|c}
\hline \multirow{3}{*}{ mode } & \multirow{3}{*}{ exact } & \multicolumn{3}{|c|}{ DB10 } & \multicolumn{3}{c}{ IN4 } \\
\cline { 3 - 8 } & & \multicolumn{3}{|c|}{ level of resolution } & \multicolumn{3}{c}{ level of resolution } \\
\cline { 3 - 8 } & & 0 & 4 & 8 & 0 & 4 & 8 \\
\hline 1 & 49.348 & 49.352 & 49.348 & 49.348 & 49.754 & 49.350 & 49.348 \\
\hline 2 & 49.348 & 66.297 & 49.406 & 49.348 & 56.131 & 49.471 & 49.348 \\
\hline \hline
\end{tabular}

For a value of $c=4 \pi^{4} E I / L^{4}$, it is expected to find both critical loads to be identical for different buckling modes. Results are in Table II.

In order to consider the beam as infinitely long, the value $c=256 \pi^{4} E I / L^{4}$ was used as the stiffness of the foundation. In this case, the critical load is the lower bound given by $P=2 \sqrt{c E I}$. Results are in Table III. The corresponding buckling mode features 4 half-waves $(n=4)$, as seen in Fig. 7.

TABLE III: RESULTS FOR AN INFINITELY LONG SIMPLY SUPPORTED BEAM ON ELASTIC FOUNDATION (IN TERMS OF $E I / L^{2}$ )

\begin{tabular}{c|c|c|c|c|c|c}
\multicolumn{4}{|c|}{ ON ELASTIC FOUNDATION (IN TERMS OF EI/L $L^{2}$ ) } \\
\hline \hline \multirow{3}{*}{ exact } & \multicolumn{3}{|c|}{ DB10 } & \multicolumn{3}{c}{ IN4 } \\
\cline { 2 - 7 } & \multicolumn{3}{|c|}{ level of resolution } & \multicolumn{3}{c}{ level of resolution } \\
\cline { 2 - 7 } & 0 & 4 & 8 & 0 & 4 & 8 \\
\hline 315.827 & 410.365 & 329.604 & 315.827 & 537.083 & 322.015 & 315.827 \\
\hline \hline
\end{tabular}

\section{2) Infinitely long beam free at both ends}

In the case of an infinitely long beam, free at both ends, subjected to an axial load (Fig. 8), the critical load has an analytical value of $P=\sqrt{c E I}$ and the buckling mode is a sinusoidal wave with increasing amplitude towards the ends of the structure. There are two possible modes: symmetric and anti-symmetric, both corresponding to the same critical load. Results for the first and second critical loads using $c=256 \pi^{4} E I / L^{4}$ are shown in table IV and the corresponding buckling modes are shown in Fig. 9.

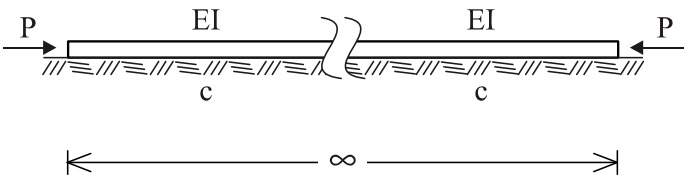

Fig. 8. Infinitely long beam free at both ends.

TABLE IV: RESULTS FOR AN INFINITELY LONG BEAM FREE AT BOTH ENDS Critical Loads $\left(E I / L^{2}\right)$

\begin{tabular}{c|c|c|c|c|c|c|c}
\hline \multirow{2}{*}{ mode } & \multirow{2}{*}{ exact } & \multicolumn{3}{|c|}{ DB10 } & \multicolumn{3}{c}{ IN4 } \\
\cline { 3 - 8 } & & \multicolumn{2}{|c|}{ level of resolution } & \multicolumn{3}{c}{ level of resolution } \\
\cline { 3 - 8 } & & 0 & 4 & 8 & 0 & 4 & 8 \\
\hline 1 & 157.91 & 180.20 & 160.89 & 157.25 & 195.06 & 159.10 & 157.25 \\
\hline 2 & 157.91 & 187.03 & 162.94 & 158.60 & 243.62 & 160.75 & 158.60 \\
\hline \hline
\end{tabular}

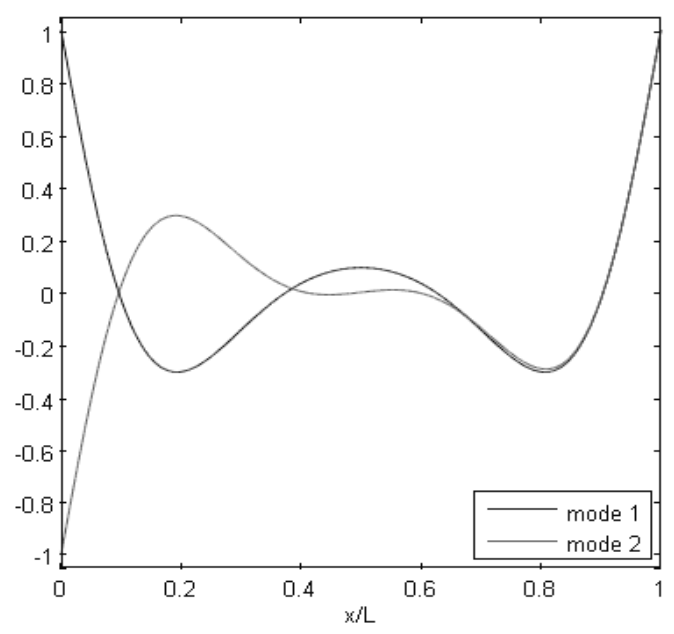

Fig. 9. Symmetric and anti-symmetric buckling modes.

It is interesting to notice that even at high levels of resolution critical loads are not accurately obtained. In all cases, the first one is lower than the exact value and the 
second one is greater.

\section{3) Other boundary conditions}

There are no analytical values for boundary conditions different from the ones studied in previous sections. For that reason, in this case results are compared with the ones obtained by standard beam finite elements. Results are shown in Table $\mathrm{V}$, where (CF) stands for clamped-free, (C) for fully clamped and (CS) for clamped and supported.

TABLE V: CRITICAL LOADS OF BEAMS ON ELASTIC FOUNDATION Critical Loads $\left(E I / L^{2}\right)$

\begin{tabular}{c|c|c|c|c|c|c|c|c|c}
\hline & \multicolumn{3}{|c|}{ DB10 } & \multicolumn{3}{c|}{ IN4 } & \multicolumn{3}{c}{ FEM } \\
\cline { 2 - 10 } & \multicolumn{3}{|c|}{ level of resolution } & \multicolumn{2}{c|}{ level of resolution } & \multicolumn{3}{c}{$\mathrm{n}^{0}$ of elements } \\
\cline { 2 - 10 } & 0 & 4 & 8 & 0 & 4 & 8 & 10 & 100 & 1000 \\
\hline $\mathrm{CF}$ & 187.7 & 162.0 & 157.9 & 218.4 & 159.9 & 157.9 & 158.4 & 157.9 & 157.9 \\
\hline $\mathrm{C}$ & 753.8 & 389.4 & 353.2 & 771.5 & 368.3 & 353.2 & 354.7 & 353.2 & 353.2 \\
\hline $\mathrm{CS}$ & 508.2 & 343.4 & 325.6 & 609.8 & 333.6 & 325.6 & 326.3 & 325.6 & 325.6 \\
\hline \hline
\end{tabular}

TABLE VI: RESULTS FOR BOUNDARY CONDITIONS (BC) AND LOADINGS (L)

\begin{tabular}{cccc}
\hline \hline $\mathrm{BC} / \mathrm{L}$ & EXACT & WGM & ERROR \\
\hline $\mathrm{C} / \mathrm{U}$ & $0.00126 q L^{4} / D$ & $0.00126 q L^{4} / D$ & $0.4 \%$ \\
\hline $\mathrm{C} / \mathrm{C}$ & $0.00560 P L^{2} / D$ & $0.00557 P L^{2} / D$ & $0.5 \%$ \\
\hline $\mathrm{S} / \mathrm{U}$ & $0.00406 q L^{4} / D$ & $0.00406 q L^{4} / D$ & $0.1 \%$ \\
\hline $\mathrm{S} / \mathrm{C}$ & $0.01160 P L^{2} / D$ & $0.01156 P L^{2} / D$ & $0.3 \%$ \\
\hline \hline
\end{tabular}

\section{Thin Plate}

Finally, to test the possibility of extending the method to two-dimensional problems, a thin plate was modeled using the equations developed in previous sections. Fig. 10 shows a

$\mathrm{M}_{\mathrm{X}} / \mathrm{P}$
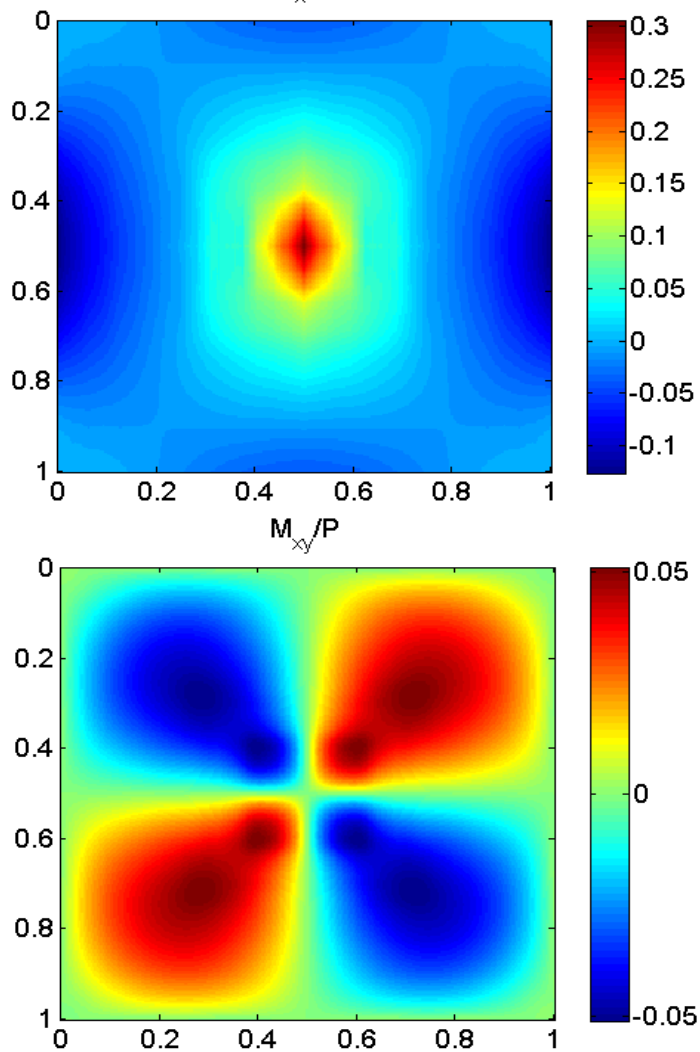

square plate with all edges clamped subjected to a concentrated load applied at its center.

The plate was modeled using the IN6 Interpolet at level 3, leading to a total number of 289 degrees of freedom. The result for the central displacement was $w=-0.00557 P L^{2} / D$ which represents an error of $0.5 \%$ when compared to the exact solution $w=-0.00560 \mathrm{PL}^{2} / D$. Results were extremely good, considering that a FE mesh using $32 \times 32$ plate elements (3267 degrees of freedom) gives an error of $0.7 \%$ in the central displacement.

Fig. 11 shows the results for displacements, bending moments $M_{x}, M_{y}$ and twisting moment $M_{x y}$. Bending and twisting moment distribution were obtained using the moment-curvature relation (second derivative of displacement). Errors in the bending moments $M_{x}$ and $M_{y}$ at the center point were approximately $4 \%$.

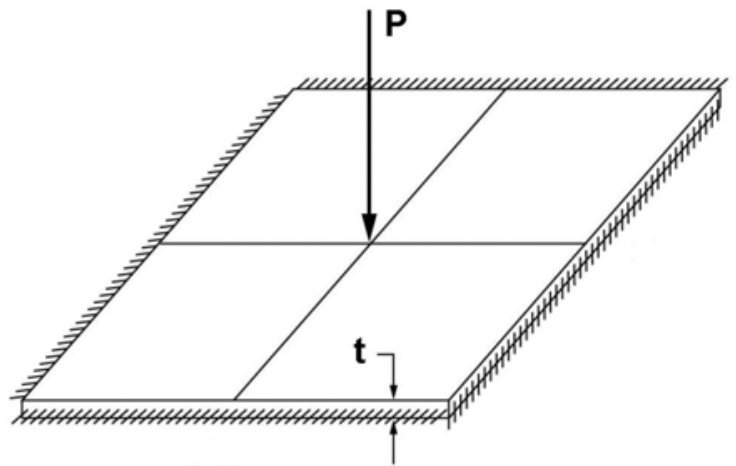

Fig. 10. Clamped plate subjected to a concentrated load at the center.
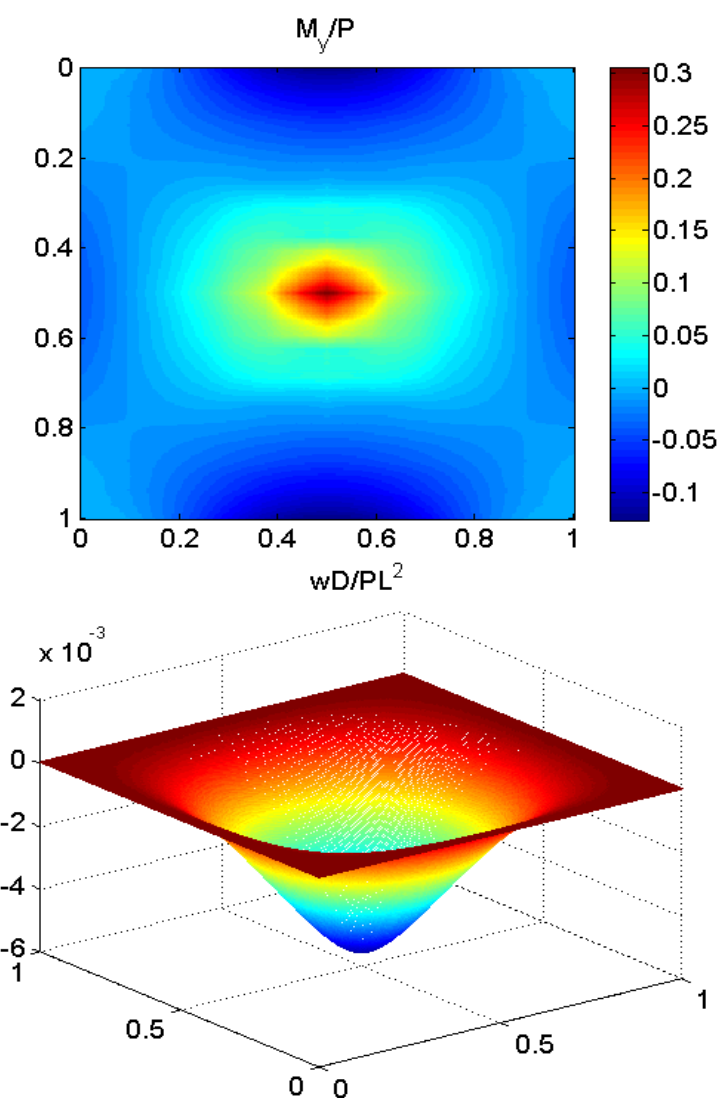

Fig. 11. Results for moments $M_{x}, M_{y}, M_{x y}$ and displacement $w$.

Different types of boundary conditions and loadings were tested for a square plate and the values obtained for central displacement are summarized in Table VI, where (U) stands for uniformly distributed loading. Results were compared 
with exact solutions given by [12].

\section{CONCLUSION}

This work presented the formulation and validation of the Wavelet-Galerkin Method using Daubechies wavelets and Deslauriers-Dubuc Interpolets. It was also shown that wavelets have the ability of capturing discontinuities without the need to place nodes where they occur.

As in the traditional FEM and other numerical methods, the accuracy of the solution can be improved either by increasing the level of resolution or the wavelet order. Sometimes, lower order wavelets at higher resolution can give better results than higher order wavelets at lower resolutions.

In the case of an infinitely long beam free at both ends, the small inaccuracy in the calculation of critical loads is probably due to mode interaction.

For two-dimensional problems, results for displacements and bending moments were extremely good, although only regular geometry problems were studied. The extension of the method to irregular geometries is still a challenge.

Since the unknowns of the method are interpolation coefficients instead of nodal displacements it is possible to obtain a smooth representation even with a reduced number of degrees of freedom.

All matrices involved can be stored and operated in a sparse form, since most of their components are null, thus saving computer resources. Due to the compact support of wavelets, the sparseness of matrices increases along with the level of resolution.

\section{REFERENCES}

[1] S. Qian and J. Weiss, "Wavelets and the numerical solution of partial differential equations," Journal of Computational Physics, vol. 106, pp. $155-175,1992$.

[2] G. Deslauriers and S. Dubuc, "Symmetric iterative interpolation processes," Constructive Approximation, vol. 5, pp. 49-68, 1989.

[3] I. Daubechies, "Orthonormal bases of compactly supported wavelets," Communications in Pure and Applied Mathematics, vol. 41, pp. 909-996, 1988.

[4] R. B. Burgos, M. A. C. Santos, and R. R. Silva, "Deslauriers-Dubuc interpolating wavelet beam finite element," Finite Elements in Analysis and Design, vol. 75, pp. 71-77, 2013.

[5] D. L. Donoho, "Interpolating wavelet transforms," Department of Statistics, Stanford University, 1992.
[6] Z. Shi, D. J. Kouri, G. W. Wei, and D. K. Hoffman, "Generalized symmetric interpolating wavelets," Computer Physics Communications, vol. 119, pp. 194-218, 1999.

[7] V. P. Nguyen, T. Rabczuk, S. Bordas, and M. Duflot, "Meshless methods: a review and computer implementation aspects," Mathematics and Computers in Simulation, vol. 79, pp. 763-813, 2008.

[8] X. Zhou and W. Zhang, "The evaluation of connection coefficients on an interval," Communications in Nonlinear Science \& Numerical Simulation, vol. 3, pp. 252-255, 1998.

[9] A. Latto, L. Resnikoff, and E. Tenenbaum, "The evaluation of connection coefficients of compactly supported wavelets," in Proc. the French-USA Workshop on Wavelets and Turbulence, pp. 76-89, 1992.

[10] X. Chen, S. Yang, J. Ma, and Z. He, "The construction of wavelet finite element and its application," Finite Elements in Analysis and Design, vol. 40, pp. 541-554, 2004.

[11] S. P. Timoshenko and J. M. Gere, Theory of Elastic Stability, McGraw Hill, New York, USA, 1961.

[12] S. Timoshenko and S. Woinowsky-Krieger, Theory of Plates and Shells, McGraw-Hill, New York, USA, 1959.

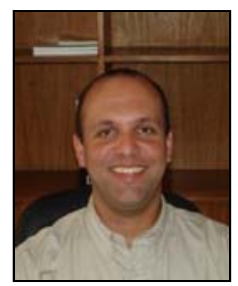

Rodrigo Bird Burgos is an assistant professor in the Department of Structures and Foundations at the State University of Rio de Janeiro. He received his B.Sc. in 2003, He received his M.Sc. in 2005 and He received his Ph.D. in 2009 in civil engineering from Pontifical Catholic University of Rio de Janeiro. Postdoctoral researcher at Pontifical Catholic University of Rio de Janeiro from 2009 to 2012

He has experience in computational modeling and advanced numerical methods for solving differential equations. His main research interests include instability and structural dynamics, wavelet functions, interpolets, Finite Element Method.

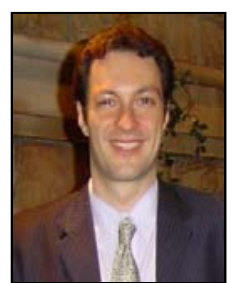

Marco Antonio Cetale Santos is an assistant professor and the head of Department of Geology and Geophysics at Fluminense Federal University. He received his B.Sc. in electronic engineering from State University of Rio de Janeiro (UERJ) in 1996 and He received his M.Sc. in 1999 and $\mathrm{He}$ received his $\mathrm{Ph} . \mathrm{D}$. in 2003 in electrical engineering from Pontifical Catholic University of Rio de Janeiro.

$\mathrm{He}$ has experience in applied geophysics and advanced numerical methods. His main research interests include signal processing, seismic inversion, seismic imaging and finite differences method.

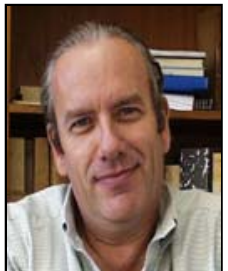

Raul Rosas e Silva is an associate professor and as the head of the Department of Civil Engineering at Pontifical Catholic University of Rio de Janeiro (PUC-Rio). He received his B.Sc. in civil engineering from Parana Federal University in 1972, He received his M.Sc. in civil engineering from PUC-Rio in 1974 and $\mathrm{He}$ received his Ph.D. in civil engineering from Stanford University in 1982.

$\mathrm{He}$ is the vice-president of Padre Leonel Franca Foundation, which manages research projects at PUC-Rio. He has experience in structural analysis and his main research interests include finite element buckling, numerical methods, constitutive models, mathematical modeling of instabilities in materials and structures. 